\title{
The most stable pinning configurations in transverse supracondylar humerus fracture fixation in children: A novel three-dimensional finite element analysis of a pediatric bone model
}

\author{
Allieu Kamara \\ Shengjing Hospital of China Medical University \\ Xianglu Ji \\ Shengjing Hospital of China Medical University \\ Chuang Liu \\ Shijiazhuang Tiedao University \\ Tianjing Liu \\ Shengjing Hospital of China Medical University \\ Enbo Wang ( $\square$ wangenbodor@163.com ) \\ Shengjing Hospital of China Medical University https://orcid.org/0000-0003-3394-2670
}

\section{Research article}

Keywords: supracondylar humerus fracture; pinning fixation; biomechanical analysis; finite element analysis; three-dimensional pediatric bone model

Posted Date: March 4th, 2020

DOI: https://doi.org/10.21203/rs.3.rs-15966/v1

License: (c) (1) This work is licensed under a Creative Commons Attribution 4.0 International License.

Read Full License

Version of Record: A version of this preprint was published at Injury on June 1st, 2021. See the published version at https://doi.org/10.1016/j.injury.2021.01.012. 


\section{Abstract}

Background : This study aimed at finding out the effect of exit height of pins, pin trajectory and pin number on the stability of crossed and lateral pinning configurations used in the fixation of extensiontype supracondylar humerus fracture (SHF) in children, through finite element analysis of a novel pediatric humerus bone model. Method : Distal humerus model consisting of the ossific nucleus of the capitellum (ONC) and distal cartilage of a 6-year-old boy was developed computationally. Various crossed and lateral pinning fixation models with either two or three pins were simulated on an extension-type, transverse SHF and tested in six loading directions. Results : Two-crossed pins and 2lateral pins were respectively more stable in rotation and compression loadings, while 3 -crossed pins were the most stable in all loading directions. The crossed pins exiting at the upper border of the distal metaphyseal-diaphyseal junction (MDJ) had the best stiffness among the 2-crossed pins, while the lateral pins with a mid-ONC distal pin provided the best stiffness among the 2-lateral pins. A third pin however, going through the olecranon fossa led to improved stability of the 2-lateral pins in flexion, extension, internal and external rotations. Conclusion : In the fixation of extension-type, transverse supracondylar humerus fractures, 2-crossed pins are only superior to 2-divergent lateral pins in rotational loadings. Two-crossed pins exiting at the upper border of the MDJ provides the best stability, whereas 2lateral pins with a distal pin going through the middle third of the ONC provides the best stability against compression forces for these fractures. Three-crossed pins however offer the best stability against both compression and rotation forces.This study offers important clues in the preoperative evaluation and management of extension-type supracondylar fractures in children.

\section{Background}

Supracondylar humerus fracture (SHF) is a commonly encountered pediatric trauma. It accounts for about $60 \%$ of all elbow fractures in children[1]. Closed reduction and percutaneous pinning is commonly used as the mainstream treatment for displaced SHFs. However, loss of reduction with the technique is a major concern to pediatric orthopedic surgeons. Causes of loss of reduction with pinning techniques have been attributed to pinning configuration, pin size, pin number and pin spread[2-7].

Crossed pins and divergent lateral pins are said to have comparable stiffness in numerous clinical and biomechanical studies[8, 9]. Many authors have however advocated for the lateral-only pins due to the risk of ulnar nerve injury with medially placed pins $[10,11]$. However, the use of crossed pins may be inevitable in some cases, such as in medially oblique fractures, high fractures or fractures with medial comminution, which may have a higher incidence of loss of reduction in clinical practice[5]. Crossed pins are also preferred by many surgeons because of its torsional stability, an important factor considered in the prevention of cubital varus[12].

Acceptable but controversial results have been shown with multiple biomechanical studies comparing crossed and divergent lateral pins[3, 9, 13-16]. However, most of those studies were heterogeneous in fixation details. Influencing factors such as the positions where the pins entered and exited the bone were 
not studied in detail. This might be one of the reasons for the different results from those studies. By using a novel pediatric bone model, our study aimed at finding out the effect of these detailed features of crossed and lateral pinning through finite element analysis.

\section{Methods}

\section{Computational modelling of the pediatric distal humerus}

A normal computed tomography (CT) data of the right elbow of a 6-year-old male child who was evaluated for occult elbow fracture, was downloaded from our hospital's picture archiving and communications systems software (PACS) in Digital Imaging and Communications in Medicine (DICOM) format after approval from our institution's Medical Ethics Committee. The data was then imported into Simpleware's image processing software (Synopsis Inc., California, USA) for data processing. This CT data was selected because no pathology was found on the elbow and also due to its clearness of the elbow cartilage profile. Segmentation of the humerus plus the ossified ossific nucleus of the capitellum (ONC) was done based on the grayscale of the CT images. Segmentation of the distal humeral cartilage was done manually according to the software's protocol. A complete triangular 3D mesh of the entire distal humerus was generated and exported in International Graphic Interactive Standard (IGES) format (Fig. 1).

\section{Pinning Fixation And Finite Element Models Generation}

The finished surface model was imported into SolidWorks 2016 edition (Dassaut Systemes-Simula, France) to generate a solid model of the distal humerus. A typical, transverse supracondylar humerus fracture, with a 20 degrees sagittal obliquity, was created at the level of the olecranon fossa, to mimic the most common fracture pattern[17]. Various crossed and lateral pinning configurations were then simulated using $1.6 \mathrm{~mm}$ diameter cylinders as Kirschner-wires, generating a total of eleven models (Fig. 2, Fig. 3). The entry points of the lower pin in the 2-lateral pinning configurations, were altered with respect to the ONC in the sagittal plane, to generate a total of 3 configurations (Fig. 3, Fig. 4). A third pin was added laterally or medially to the stiffest 2-lateral pinning configuration (2LCap) after testing the lateral pins, to give the 3-pinning configurations 3LLC, 3LOF and 3CP (Fig. 2). The fixated models were imported into HyperMesh14 (Altair Engineering, Michigan, USA) for pre-processing and meshing. Material properties for the finite element analysis were assigned according to Table $1[18,19]$.

\section{Finite Element Calculation And Determination Of Stiffness}

The finished meshed models were imported into Abaqus 6.14 (Dassaut Systemes-Simula, France). Near the position of the established geometric center, a node was selected as a reference point at the loading surface. A coupling constraint was then set between the loading surface and the reference point. The proximal end of the humerus was completely restrained and a concentrated force of $30 \mathrm{~N}$ was applied to 
the reference point along the $X,-X$ axes and $Y,-Y$ axes to simulate flexion, extension, valgus and varus loadings. A torsional moment of $1.5 \mathrm{~N} \mathrm{~m}$ was also applied along the $Z$ and $-Z$ axes to simulate internal and external rotations. The maximum displacement of the distal fragments relative to the proximal fragments were recorded and the corresponding stiffnesses were computed.

\section{Stress Analysis On Pins}

In order to determine the technique that generated the most stress on pins, we recorded and analyzed the stress distribution regions and levels on pins, and also determined the highest stress levels on individual pins in each technique, in order to determine the pin that contributed more to the stability of techniques.

\section{Results}

We compared the stiffness of crossed pins at different exit points. The CP4 crossed pinning configuration, in which the pins exited at the upper border of the metaphyseal-diaphyseal junction (MDJ), had the best stiffness in all directions. A further increase or decrease in exit height did not result in any further increase in stiffness (Fig. 5a). Among the 2-divergent lateral pinning configurations, stiffness was better when the distal pin entered through the ONC. The 2LCap configuration which had its distal pin going through the middle third of the ONC, had the best stiffness (Fig. 5). Overall, 2LCap had the best stiffness in compression tests, while CP4 had the best stiffness in rotational tests when all the two pins configurations were compared (Fig. 5b).

When an additional pin was used with the 2LCap configuration, its overall stiffness significantly improved in all directions. The 3-crossed pinning configuration $3 \mathrm{CP}$, had the best overall stiffness among the 3pinning configurations. Stiffness more than tripled in both coronal and rotational loadings and doubled in sagittal loadings (Fig. 5b). The 3-lateral configuration 3LLC, which had its third pin lying within the lateral column was only stiffer than 3LOF in sagittal loadings while the 3-lateral configuration 3LOF which had its third, middle pin going through the olecranon fossa was stiffer in coronal and rotational loadings.

When the 3-pins configurations were compared with the best 2-pins configurations, 3CP was still the stiffest in all directions, followed by the 3-lateral pins configurations in compression tests and CP4 in rotational tests. Stress analysis showed the highest stress levels generated during rotational tests to be located on the medial pins which means that, medial pins play a crucial role in maintaining torsional stability of crossed pins. In the divergent lateral pinning configurations, the distal (capitellar) lateral pins experienced the highest stress levels individually, also demonstrating their contributing role in the stability of lateral pins.

\section{Discussion}

Biomechanical studies via computational finite element simulation can provide deeper insight into the stability and functionality of bone constructs, and therefore can be used to make comparisons among 
various fixation techniques that are difficult to be accomplished through other methods[20]. This study is the first to establish a pediatric bone model for three-dimensional finite element analysis through a novel combination of commonly available commercial software. In this model, we compared the stiffness of prevalent Kirschner wire configurations and the variations in fixation patterns of supracondylar fracture of the humerus. We observed that 2-crossed pins are not stiffer than 2-divergent lateral pins in some circumstances, as it used to be believed by many surgeons. A third pin going through the olecranon fossa, inserted through the ossific nucleus of the capitellum substantially enhanced the stiffness of 2divergent lateral pins configuration.

Previous finite element analytic studies on pediatric fractures exclusively utilized synthetic bone models[14]. The dramatic difference in the anatomy of the distal humerus between adults and children would inevitably lead to divergence from clinical reality. In this study, through a combination of of innovative software packages, we established a pediatric humerus model with the computed tomography images of a 6-year-old boy, in which the child-characteristic cartilaginous component and the ossific nucleus of the capitellum were reconstructed for the finite element analytic tests.

Among all the pinning configurations, divergent lateral pins were more resistant than crossed pins to translational forces. Crossed pins were however more resistant to rotational forces. The stiffness values of the pinning configurations were mainly influenced by the entry and exit points of pins, pin pathway and number of pins. These findings may partly explain the different conclusions of previous studies, since most of those studies differed in the entry or exit points of pins.

It is mostly believed that, the more proximal pins exit, the more stable the fixation would be. In our crossed pinning configurations however, the best stiffness was achieved when both pins exited around the upper border of the metaphyseal-diaphyseal junction (MDJ). This superiority was most obvious in the anti-rotation ability. It had been reported that proper pin spread at the fracture site could influence the stability of pins[5]. We observed from our study that, by exiting around the upper border of the MDJ in typical SHFs, the ideal pin spread could be achieved and therefore optimum stability may be obtained. Although the higher the crossed pins exited the more pin spread achieved along the fracture line, stiffness however reduced as they exited higher. This was probably because the center of rotation was brought closer to the crossing point as the pins exited higher, thereby making them less stable. Pins that crossed within the MDJ region would have their centre of rotation brought closer to the fracture line, also making them less stable. Similar observation was made with crossed pins in a previous biomechanical study conducted with composite bones using varying fracture heights of metaphyseal-diaphyseal fracture of the humerus[21].

The entry point and pin pathway had a major influence on the total stiffness of lateral pins. One pin entering through the ONC greatly enhances the stiffness of divergent lateral pins in all aspects. When entering from the ONC, there is more pin-cortical bone contact area, rendering pins more stability. This finding was consistent with that of Gottschalk et al,[3]. The distal lower pins of the two lateral-entry configurations (2LP, 2LD) in our model, with only a small or no ONC contact area, may have to penetrate 
and re-enter the bone through their course along the olecranon fossa giving them a shorter bone tunnel length and therefore less stability. Our study was however not the first to emphasize on the effect of lateral pin pathways. Using synthetic bone model, one study compared the influence of the distal lateral pin when the position of the other, proximal lateral pin was fixed. They came to the conclusion that, a lateral pin placed parallel to the metaphyseal flare of the lateral humeral cortex, in combination with a second diverging pin crossing the fracture site at the medial edge of the coronoid fossa, provides the optimum fixation[22]. It was however not clear whether the second pin was a capitellar entry one.

Adding a third pin to 2-divergent lateral pins in any configuration significantly enhances stiffness of lateral pins in all directions. From our study, we observed that a third, middle pin travelling all the way within the bony cavity of the lateral column increases stability of lateral pins in the sagittal plane, while that going through the olecranon fossa increases stability in the coronal and rotational planes. We believe the increased stability demonstrated is due to the more reinforcement along the fracture line by the additional pin, longer bone tunnel length and more pin-cortical bone contact regions. The best stiffness was however generated by the 3-crossed pin configuration (3CP, 2-divergent lateral and 1-medial pins), which had a mid-ONC distal lateral pin. Stiffness values were far more than any other 3-pins configuration in all loading directions. Our finding differed from that of Feng $\mathrm{C}$ et al., who stated that an additional pin did not bring about increased stiffness in their model[6]. This difference may be partly due to the difference in testing models, fracture patterns and entry and exit points.

Despite the novelty of this model, our study had some limitations. First, the model was based on the computed tomography images of a 6-year-old boy, which may not represent the diverse pediatric population. However, since the majority of SHFs occur around this age, it was therefore used for our model. Secondly, we only looked at the most commonly encountered typical, transverse fracture pattern[17], which may not represent all clinical circumstances of pediatric supracondylar humeral fractures. Models of different age, gender and fracture pattern must be developed and tested, as well as clinical observations should be carried out to further verify the findings in this study.

\section{Conclusion}

This study demonstrated the influence of entry point, exit point, pin number and pathway on the stiffness of Kirschner wire fixation in pediatric transverse supracondylar humerus fractures. It offers important clues in the preoperative evaluation and management of these fractures. Compared to 2-crossed pins, 2lateral pins with a mid-capitellar distal pin in maximum divergence would provide better stability against translational forces for satisfactorily reduced transverse fractures. Three-divergent lateral pins are however more stable than 2-divergent lateral pins and would be recommended in cases of unstable reduction. Despite the risk of ulnar nerve injury, crossed pins still provide the best stability against rotational forces with pins exiting at the upper border of the MDJ providing the best stability. For optimum stability against both translational and rotational forces, two-divergent lateral pins (with a midcapitellar distal pin) and one medial pin is recommended for transverse supracondylar humerus fractures. If there is however any concern for ulnar nerve injury with medial pins, 3-divergent lateral pins 
with at least one mid-capitellar distal pin can offer comparable stability as crossed pins and should be used as an alternative pinning configuration

\section{List Of Abbreviations}

SHF: Supracondylar humerus fracture

ONC: ossific nucleus of the capitellum

MDJ: Metaphyseal-Diaphyseal Junction

CP: 2-crossed pins

3CP: 3-crossed pins

3LLC: 3-lateral pins with middle pin lying within the lateral column

3LOF: 3-lateral pins with middle pin traversing the olecranon fossa

2LCap: 1-direct lateral and 1-direct capitellar entry pins

2LP: 1-direct lateral and 1-posterior capitellar entry pins

2LD: 2-direct lateral entry pins

\section{Declarations}

\section{Ethics approval and consent to participate}

This research was approved by the Medical Ethics Committee of Shengjing Hospital of China Medical University (reference number 2018PS361K). We obtained verbal consent from the participant's parents for the CT data to be used in our study. Obtaining of verbal consent was also approved by the Medical Ethics Committee of Shengjing Hospital of China Medical University.

\section{Consent for publication}

Not applicable.

\section{Availability of data and materials}

The datasets used or analysed during the current study are available from the corresponding author on reasonable request.

\section{Funding}


There was no funding for this work.

\section{Authors' Contributions}

$\mathrm{AK}, \mathrm{XJ}, \mathrm{TL}$ and EW designed the fracture and internal fixation models. CL computationally established the three-dimensional pediatric humerus model and also completed the finite element analysis. AK completed statistical analysis, initial drafting of manuscript and editing work. XJ and TL completed some data processing work, redrafted and edited part of this manuscript. EW edited and revised the manuscript critically for important intellectual content and approval of final version for publication. All authors participated in every revision and improvement of the manuscript and read and approved the final manuscript.

\section{Competing interests}

The authors declare that they have no competing interests.

\section{Acknowledgment}

The authors thank the Department of Radiology, Shengjing Hospital of China Medical University for their assistance in obtaining the raw CT data, as well as the anonymous reviewers and copy editor for their valuable comments proposed.

\section{References}

1. Houshian S, Mehdi B, Larsen MS. The epidemiology of elbow fracture in children: analysis of 355 fractures, with special reference to humerus fractures. J Orthop Sci. 2001;6:312-315.

2. Aarons CE, Lobts $C$, Chan DB, et al. Repair of supracondylar humerus fractures in children: does pin spread matter? J Pediatr Orthop B. 2012;21(6):499-504.

3. Gottschalk HP, Sagoo D, Glaser D, et al. Biomechanical analysis of pin placement for pediatric supracondylar humerus fractures: does starting point, pin size, and number matter? J Pediatr Orthop. 2012;32(5):445-451.

4. Pennoc AT, Charles M, Moor M, et al. Potential causes of loss of reduction in supracondylar humerus fractures. J Pediatr Orthop. 2014;34(7):691-7.

5. Reisoglu A, Kazimoglu C, Hanay E, et al. Is pin configuration the only factor causing loss of reduction in the management of pediatric type III supracondylar fractures? Acta Orthop Traumatol Turc. 2017; 51(1):34-38.

6. Sankar WN, Hebela NM, Skaggs DL, et al. Loss of pin fixation in displaced supracondylar humeral fractures in children: causes and prevention. J Bone Joint Surg Am. 2007;89(4):713-717.

7. Srikumaran U, Tan EW, Erkula G, et al. Pin size influences sagittal alignment in percutaneously pinned pediatric supracondylar humerus fractures. J Pediatr Orthop. 2010;30(8):792-798. 
8. Feng C, Guo Y, Zhu Z, et al. Biomechanical analysis of supracondylar humerus fracture pinning for fractures with coronal lateral obliquity. J Pediatr Orthop. 2012;32(2):196-200.

9. Weinberg AM, Castellani $C$, Arzdorf $M$, et al. Osteosynthesis of supracondylar humerus fractures in children: a biomechanical comparison of four techniques. Clin Biomech (Bristol, Avon). 2007;22(5):502-9.

10. Dekker AE,Krijnen P, Schipper IB. Results of crossed versus lateral entry K-wire fixation of displaced pediatric supracondylar humeral fractures: a systematic review and meta-analysis. Injury. 2016;47:2391-2398.

11. Prashant K, Lakhotia D, Bhattacharyya TD, et al. A comparative study of two percutaneous pinning techniques (lateral vs medial-lateral) for Gartland type $\otimes$ pediatric supracondylar fracture of the humerus. J Orthop Traumatol. 2016;17:223-229.

12. Krusche-Mandl I, Aldrian S, Köttstorfer J, et al. Crossed pinning in paediatric supracondylar humerus fractures: a retrospective cohort analysis. Int Orthop. 2012;36(9):1893-1898.

13. Bloom T, Robertson C, Mahar AT, et al. Biomechanical analysis of supracondylar humerus fracture pinning for slightly malreduced fractures. J Pediatr Orthop. 2008;28:766-772.

14. Lamdan R, Liebergall M, Gefen A, et al. Pediatric supracondylar humerus fractures: effect of boneimplant interface conditions on fracture stability. J Child Orthop. 2013;7(6):565-569.

15. Larson L, Firoozbakhsh K, Passarelli R, et al. Biomechanical analysis of pinning techniques for pediatric supracondylar humerus fractures. J Pediatr Orthop. 2006;26:573-578.

16. Vlahović T, Bumci I. Biomechanical evaluation of the value of osteosynthesis in supracondylar fracture of the humerus using Kirschner pins in children. Eur J Pediatr Surg. 2002;12:410-415.

17. Bahk MS, Srikumaran U, Ain MC, et al. Patterns of pediatric supracondylar humerus fractures. J Pediatr Orthop. 2008; 28(5):493-499.

18. Meng Y, Pak W, Guleyupoglu B, et al. A finite element model of a six-year-old child for simulating pedestrian accidents. Accid Anal Prev. 2017;98:206-213.

19. Xu M, Qu W, Wang Y, et al. Theoretical Implications of Periacetabular Osteotomy in Various Dysplastic Acetabular Cartilage Defects as Determined by Finite Element Analysis. Med Sci Monit. 2016;26;22:5124-5130.

20. Benli S, Aksoy S, Havltcloglu H, et al. Evaluation of bone plate with low stiffness material in term of stress distribution. J Biomech. 2008;41:3229-3235.

21. Kamara A, Ji X, Liu T, et al. A comparative biomechanical study on different fixation techniques in the management of transverse metaphyseal-diaphyseal junction fractures of the distal humerus in children. Int Orthop. 2019;43(2):411-416.

22. Hamdi A, Poitras $P$, Louati $H$, et al. Biomechanical analysis of lateral pin placements for pediatric supracondylar humerus fractures. J Pediatr Orthop. 2010;30:135-139.

\section{Table 1}




\begin{tabular}{|c|c|c|c|c|c|}
\hline Part & Element Type & Number of element & Number of nodes & Young's modulus (MPa) & Poisson ratio $(\lambda)$ \\
\hline Cortical bone & C3D6 & 94592 & 68822 & 11670 & 0.28 \\
\hline Cancellous bone & C3D4 & 255931 & 56916 & 70 & 0.2 \\
\hline Ossific nucleus & C3D4 & 36328 & 7864 & 70 & 0.2 \\
\hline Cartilage & C3D4 & 169888 & 36627 & 15 & 0.45 \\
\hline Two K-wires & C3D8R & 4384 & 6072 & 200000 & 0.33 \\
\hline Three K-wires & C3D8R & 5952 & 8250 & 200000 & 0.33 \\
\hline
\end{tabular}

Table 1. Material properties, number of elements and nodes of the finite element models

\section{Figures}
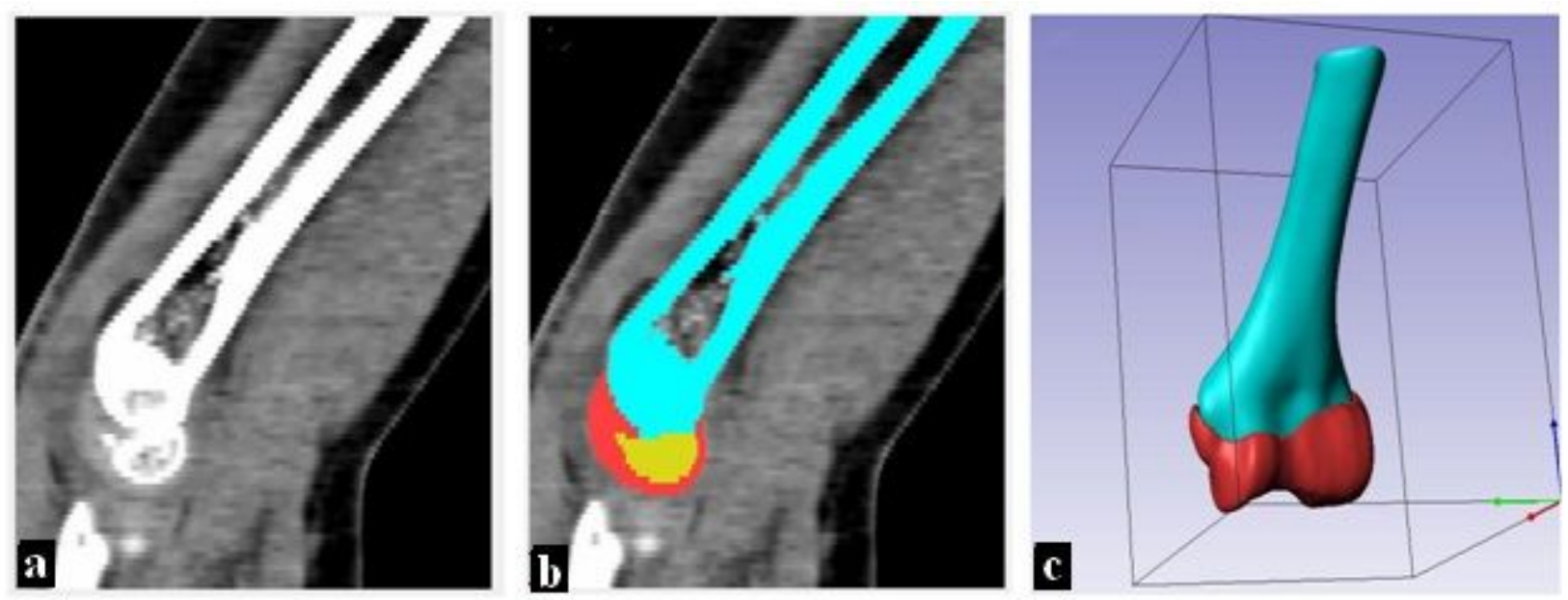

Figure 1

Simpleware interface of the reconstructed distal humerus a. 2D sagittal view of the chosen elbow from the CT images b. 2D sagittal view of the reconstructed distal humerus c. 3D view of the distal humerus. The distal cartilage, ONC and distal humerus are respectively shown in red, yellow and blue on images $b$ and $\mathrm{c}$ 


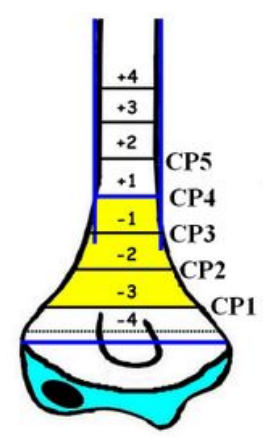

Exit points for crossed pins

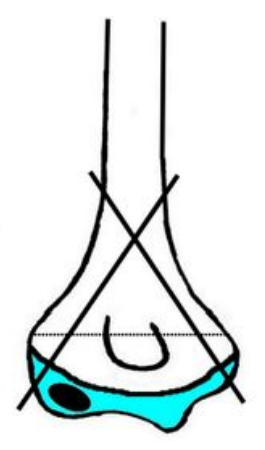

2-crossed pins

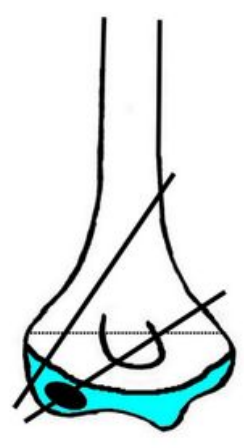

2-lateral pins

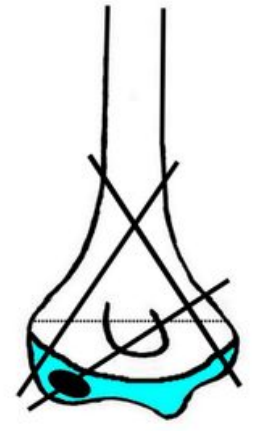

3-crossed pins (3CP)

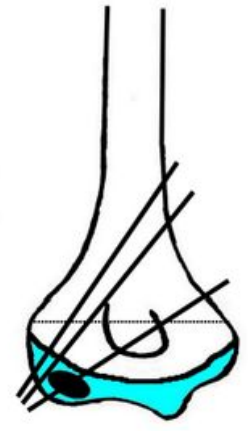

3-lateral pins (3LLC)

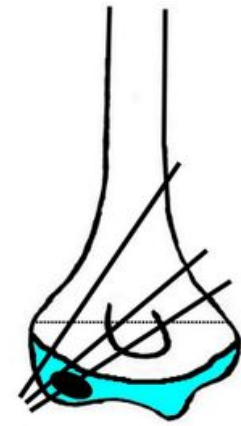

3-lateral pins (3LOF)

Figure 2

Pinning techniques used in the simulation tests. The exit zones of the 2-crossed pins were determined by dividing the regions below and above the upper border of the metaphyseal-diaphyseal junction (shaded yellow) into equal zones ( -4 to +4$)$, based on the distance from the upper border (upper blue line) to the inter-epicondylar line (lower blue line). The medial pin of the 2-crossed pins entered from the center of the medial epicondyle, while the lateral pin entered directly at the same level as the medial pin entry point. Both pins exited at the lower borders of $-3,-2,-1,+1$ and +2 zones for CP1, CP2, CP3, CP4 and CP5 respectively. CP: 2-crossed pins; 3CP: 3-crossed pins; 3LLC: 3-lateral pins with middle pin lying within the lateral column; 3LOF: 3-lateral pins with middle pin traversing the olecranon fossa

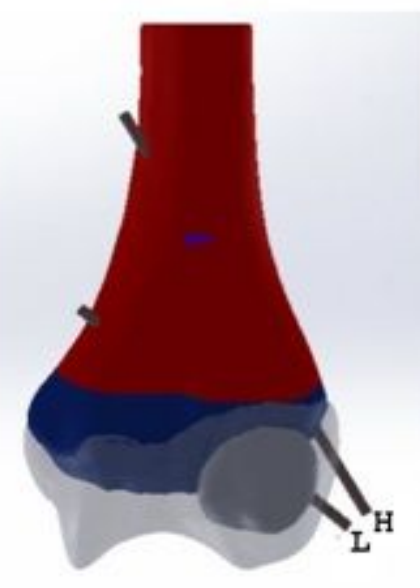

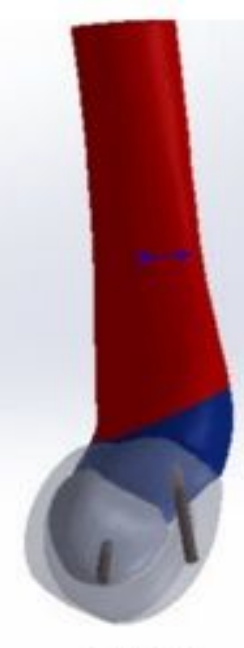

2LCap

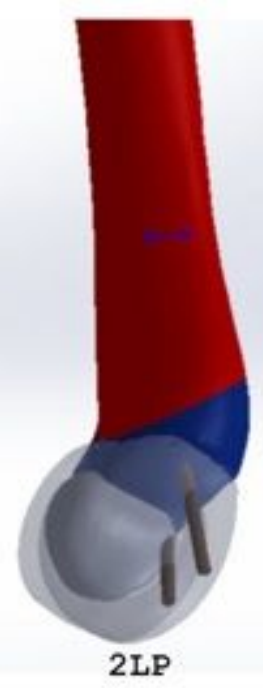

2LP

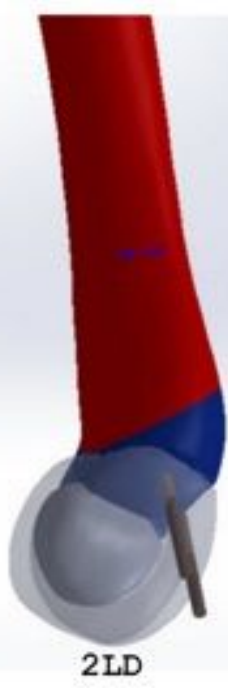

2LD

\section{Figure 3}

The 2-lateral pinning configurations. H: higher lateral pin. L: lower lateral pin. The position of Pin $\mathrm{H}$ was fixed in both coronal and sagittal planes, while the position of pin $L$ was fixed in only the coronal plane but varied with respect to the ONC in the sagittal plane. 2LCap (1-direct lateral and 1-direct capitellar entry pins), pin L lies in the middle third of the ONC; 2LP (1-direct lateral and 1-posterior capitellar entry pins), pin $L$ lies in the posterior third of the ONC; 2LD (2-direct lateral entry pins), pin L lies posterior to the ONC. ONC: the ossific nucleus of the capitellum 


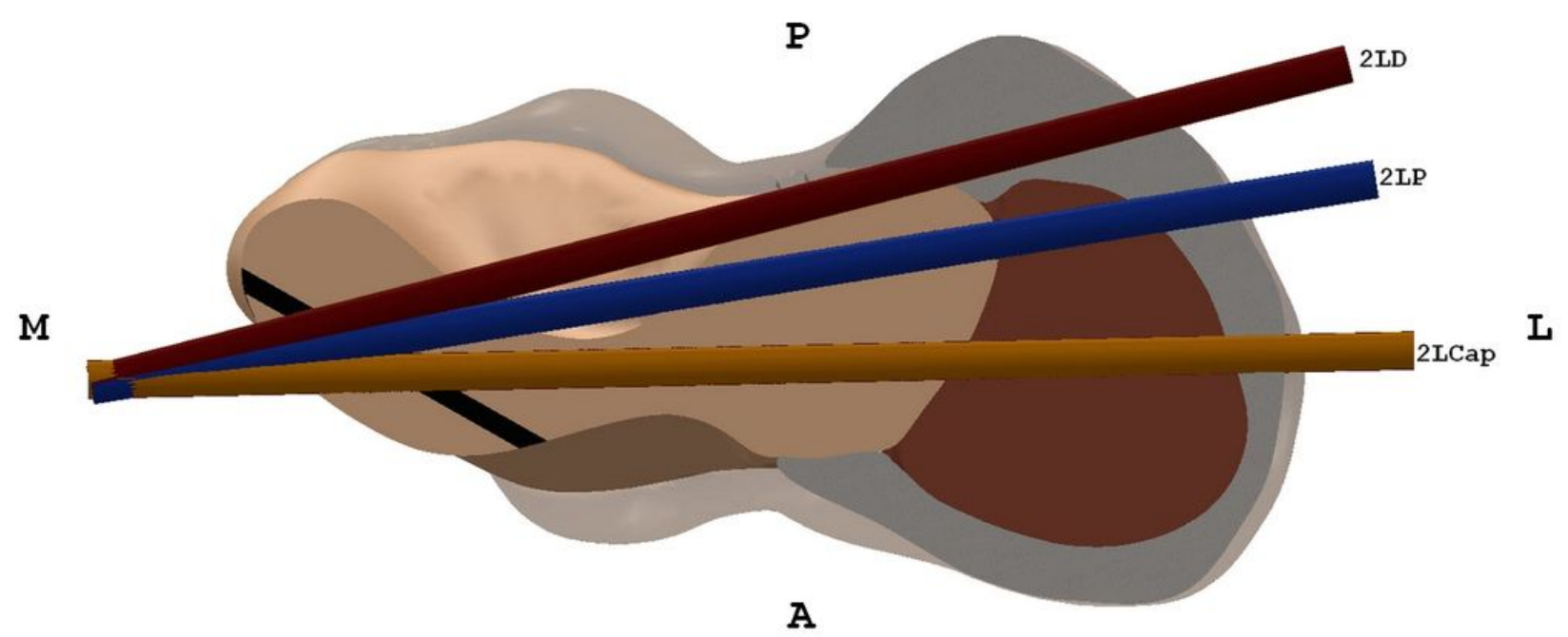

Figure 4

An axial cross-sectional view of the distal humerus showing trajectories of the lower pins in the 2-lateral pinning configurations. The lower pins of 2LCap and 2LP trisected the middle third and posterior third of the ONC respectively, while the lower pin of $2 \mathrm{LD}$ lied posterior to the ONC. The thick black line is a portion of the fracture line. ONC: ossific nucleus of the capitellum; A: anterior; P: Posterior; M: Medial; L: Lateral
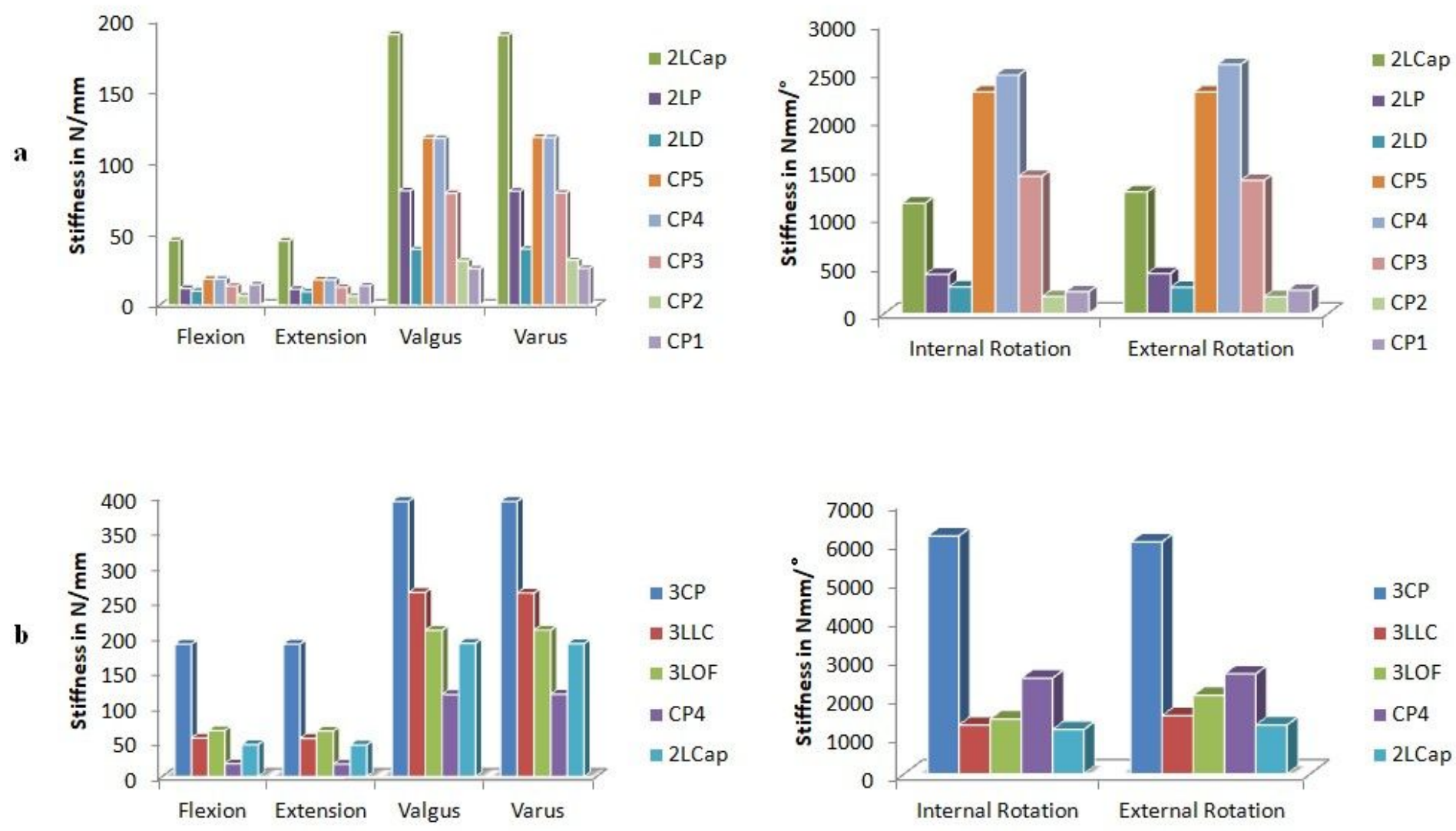

Figure 5 
Stiffness values for pining configurations a. Two-pin configurations. CP4 and 2LCap were respectively stiffest among the crossed pins and lateral pins. In compression tests, 2LCap was stiffer than all crossed pins while CP4 was stiffer than all lateral pins in rotation tests. b. Three-pin configurations compared with the best 2-pin configurations. 3CP was the stiffest in all directions, followed by $3 \mathrm{LOF}$ in flexion and extension, 3 LLC in valgus and varus and CP4 in internal and external rotations 